V.

\title{
Jahresbericht über die Tätigkeit der Ohren- und Halsklinik des Kommunehospitals zu Kopenhagen im Jahre 1907.
}

(Direktor: Prof. Dr. Holger Mygind.)

Von

Dr. Jörgen Möller, Priatdozent u. I. Assistenzarzt der Klinik.

In der Poliklinik wurden während des Jahres 19071360 Patienten behandelt, die Zahl der Konsultationen betrug 10,589 .

In der stati onären Klinik war der Krankenbestand am 1. Januar 1907 : 2 Männer, 1 Frau und 6 Kinder, neu aufgenommen wurden 311 Kranke und zwar 103 Männer, 86 Frauen und 122 Kinder. Entlassen wurden 102 Mănner, 81 Frauen, 119 Kinder, im ganzen 302, gestorben sind 3 Männer 4 Frauen, 5 Kinder, also 12, demnach eine Mortalität von 3,2\%. Der Krankenbestand am 1. Januar 1908 betrug 2 Frauen und 4 Kinder, im ganzen 6.

Der durchschnittliche Krankenbestand war 12,4 und die durchschnitt. liche Anzahl Verpflegungstage der 320 behandelten Patienten 14,7. Etatsmäßig sind in der Klinik 23 Betten vorhanden, die jedoch der Ohren- und Augenklinik gemeinschaftlich sind (außerdem kann die Klinik nötigenfalls über ein oder mehrere der den verschiedenen Abteilungen gemeinschaftlichen Einzelzimmer für Patienten erster Klasse verfügen); ein paar Betten können zwar noch Raum finden, jedoch reicht es bei weitem nicht gegen den großen Andrang von Patienten (wie aus der folgenden Übersicht hervorgeht) wurden während des Jabres nicht weniger als 109 Mastoidoperationen vorgenommen), weshalb die Patienten so schnell als möglich entlassen werden müssen, wie auch aus der auffallend kleinen Zahl der Verpflegungstage erhellt; wenn keine besonderen Komplikationen vorhanden sind, werden denn auch die Mastoid-Operierten meistens schon am Tage des ersten Verbandwechsels entlassen, also am 7.-9. Tage nach der Operation. Bisher haben wir keine üblen Zufälle als Folge dieses stark abgekürzten Klinikaufenthaltes erlebt, jedoch wäre es sehr wünschenswert, wenn die Klinik in irgend welcher Weise über eine größere Bettenzahl verfügen könnte, sodaß sich die Patienten nötigenfalls einige Zeit nach der Operation erholen könnten, auch könnte man dann viele andere Fälle in der Klinik behandeln, die jetzt nichí aufgenommen werden können, indem die operativen Fälle fast alle Plätze in Anspruch nehmen: z. B. wäre es von großer Bedeutung eine Anzahl von akuten unkomplizierten Mittelohreiterungen stationär behandeln zu können.

Als Assistenten fungierten: Dr. Jörgen Möll er, Dr. P. Tetens Hald und Dr. J. Hempel; als Volontärassistent ferner Dr. C. Speyer.

Übersicht über die wichtigsten der im Jahre 1907 in der Poliklinik wegen Ohren- und Nasenkrankheiten vorgenommenen Operationen.

Parazentese des Trommelfelles . . . . . . 37

Extraktion von Polypen und Granulationen . 18

Extraktion von Fremakörpern im Gehörgang 3 


Endonasale Operationen
Entfernung adenoider Vegetationen
Tonsillotomie

Übersicht über die wichtigsten im Jahre 1907 in der Station behandelten Ohren- und Nasenkrankheiten.

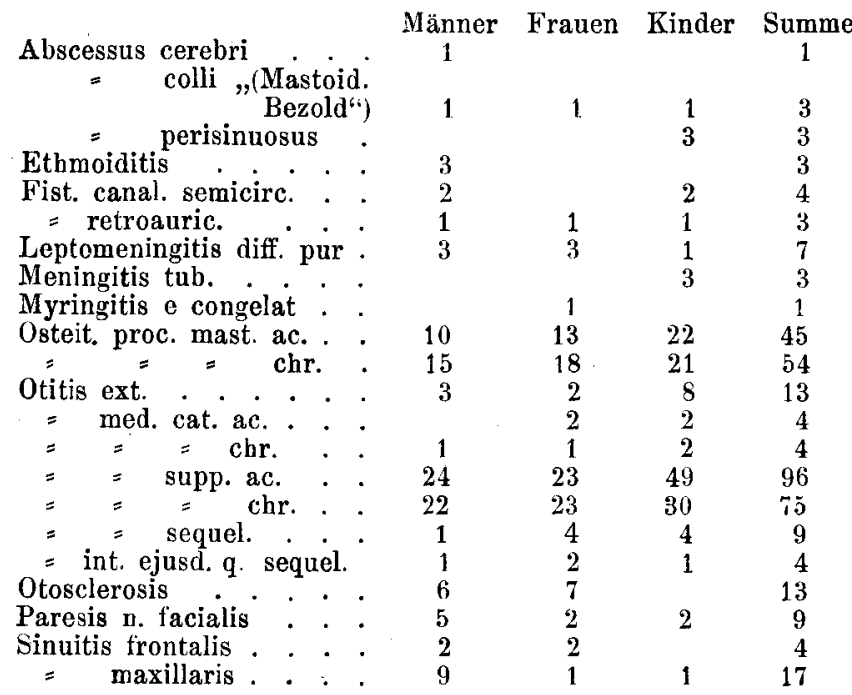

Übersicht über die im Jahre 1907 in der Station wegen Ohrenund Nasenkrankbeiten vorgenommenen Operationen.

Conchotomia

Craniotomia

Excisio partialis membr. tymp. 15 Punctio lumbalis : $: \vdots:{ }^{\circ} 17$

Excisio synechiae cavi nasi . . I Resectio aur. med. total. . . . 58

Excisio tumoris cyst. cavi nasi. 1 Resectio proc. mast. Schwartze 42

Excochleatio fist. retroaur. . . 2 Resectio proc. mast. atyp. . . 9

Extractio corp. al. aur. . . . 1 Resectio cristae septi nasi. . . 1

Extractio corp. al. cavi nasi . . 1 Resectio septi nasi submucosa. 3

Extractio granulation. aur. . . 2 Resectio sin. maxill. . . . . . 3

Extractio polypi aur: . . . . 3 Resectio sin.maxill. Luc-Caldwell 5

Extractio polypi nasi . . . . 2 Resectio sin. frontal. . . . . 1

Incisio cerebri . . . . . . 8 Resectio sin. frontal Killian . . 1

Incisio membr. tymp. . . . . 43 Trepanatio sin max. . . . 3

Incisio retroaur. c. extr. corp. al. 1 Trepanatio sin. frontalis . . . 1

\section{Übersicht über die letalen Fälle.}

Unter den 12 Gestorbenen sind nur die 6 an otogenen Komplikationen gestorben. Die Todesursachen der anderen waren: Cancer oesophagi 1, Bronchopneumia 2, Pneumonia crouposa 1, Tuberkulöse Meningitis 2. Da die letzten 2 Fälle auch otologisches Interesse darbieten, werden sie unten mit den Fällen von otogenen Komplikationen năher besprochen. Von den Gestorbenen waren 7 einer Mastoidoperation unterzogen worden, was also bei 109 Operationen eine Mortalităt von $6,3 \%$ gibt. 
I. A nna Elisabeth P., 65 Jahre, aufgenommen 10. Mărz 1907, gest. 12. Febr. 07 .

Klinische Diagnose: Otitis med. sup. ac. sin. - Leptomeningitis diff. purul. - Sektionsdiagnose: Mening. purul. diff. - Otit. pur. sin. - Bronchopneum. sin.

Anamnese: Patientin kam am 21. Febr. in die Poliklinik zur Behandlung; sie hatte damals seit 3 Tagen Ohrschmerzen links und Ausfluß. Das Trommelfell war gerötet und geschwollen, in der Mb. flacc. eine Perforation mit geschwollenen Rändern, ferner eine kleine Perforation nach hinten in der $\mathrm{Mb}$. tensa. Während der folgenden Tage wurden Röte und Schwellung geringer und obwohl sie etwas febril war, befand sie sich im ganzen recht wohl; es wurde ihr am 7. März vorgeschlagen, sich in die Station aufnehmen zu lassen, indem man wegen des Fiebers und wegen der vorwiegenden Lokalisation der Eiterung zum Kuppelraum eine Retention befürchtete, sie verweigerte es aber. Am 10. März wurde sie alsdann ohne irgend welche Aufklärung in völlig bewußtlosem Zustand in die Klinik eingeliefert.

Status praesens: Patientin ist bewußtlos, reagiert nur sehr schwach auf lautes Zurufen, reagiert heftig gegen jede Untersuchung. Nackensteifigkeit vorbanden, "Kernig". angedeutet, kein ,Trousseau". Linkes Trommelfell rot, geschwollen; kaum fühlbare Schwellung der Warze. Durch Lumbalpunktur wird $2 \mathrm{ccm}$ trübe Flüssigkeit entleert. Temp. 4॥,7.

Trotz des desolaten Zustandes wird, da laut der Anamnese die vorhandene Meningitis eine ganz frische sein muß, Totala ufmeißelung vorgenommen. Es wird eine ziemlich ausgedehnte akute Ostitis vorgefunden, die bis an die Dura reicht. Keine Labyrinthfistel. Die Dura wird entblößt und gespalten, wobei nur eine geringe Menge Flüssigkeit herausfließt. Probeinzision des Hirns ergab keinen Eiter.

Der Zustand blieb nach der Operation derselbe. Am 12. wurde wiederum Lumbalpunktur vorgenommen; nachmittags Exitus.

Mikroskopie der Cerebrospinalflüssigkeit: Zahlreiche Zellen, überwiegend polynukleäre Leukozyten, doch auch einige Lymphozyten. Bakteriologische Untersuchung: Reinkultur eines lanceolaten, mit Kapselversehenen Diplokoccus, der auch im Wachstumsmodus dem Fränkelschen ähnlich ist.

A uszug aus dem Sektionsprotokoll: Keine Thrombose der venösen Sinus. Pia überall ödematös, mit Eiter infiltriert, am meisten an der Schädelbasis. In der Schädelhöhle ca. $20 \mathrm{ccm}$ sero-purulente Flüssigkeit.

Epikrise: Während die meisten Fälle von otogener Meningitis wohl solche sind, in denen das primäre Ohrleiden erst spät zur ärztlichen Behandlung kommt, haben wir es hier mit einer Mittelohreiterung zu tun, die anscheinend rechtzeitigr zur Behandlung gelangte und die auch in normaler Weise zu verlaufen schien. Da jedoch nach 17 Tagen die Eiterung nicht recht abnehmen wollte und Patientin fortwährend etwas febril war, wurde ihr Hospitalaufnahme vorgeschlagen, sie verweigerte sie aber. 3 Tage später wurde sie in schwer komatösem Zustande in die Klinik eingeliefert und als letzter Rettungsversuch wurde sofort Aufmeißelung und Kraniotomie in Verbindung mit Lumbalpunktur vorgenommen, sie ist aber am nächstfolgenden Tag gestorben.

2. A malie D.. 47 Jahre, aufg. 28. März 07, gest. 30. März 07.

Klinische Díagnose: Otitis med supp. ac. d. in chr. - Leptomeningitis diff. purul. - Albuminuria. 
Sektionsdiagnose: Leptomeningit. pur. diff. - Otit. med. supp.

Anamnese: Es scheinen mehrmals Ohrschmerzen und Ausfluß vorhanden gewesen zu sein, diese Symptome sind jedoch nicht beachtet worden. Vor etwa 5 Tagen Kopfschmerzen und rechtsseitiger Ohrflub, konnte jedoch vor 4 Tagen einen Spaziergang auf 1 Stunde vornehmen. Die letzten 3 Tage bettlägerig. Keine Schwindelerscheinungen. Heute Nacht mehrmals Erbrechen, heute morgen etwas benommen.

Status praescens: Patientin ist sehr unrubig, beugt und streckt in einem fort die Beine, klăgelt fortwährend. Sie beantwortet fast alle Fragen vernünftig - wenn sie überhaupt antwortet - 'Temp. 39.5 Puls, 100, regelmăßig, kräftig. Keine Paresen, Pupillen kontrahiert, reagieren gut. A ur d.: Geringe Menge nicht riechenden Eiters, Trommelfell rot, geschwollen, einige Druckempfindlichkeit der Warze, vielleicht auch geringe Schwellung. Keine ausgesprochene Nackensteifigkeit, ,Kernig“" und Trousseau vorhanden. Da sie sich bei den Vorbereitungen zur Lumbalpunktur höchst ungebärdig zeigt, wird dieselbe verschoben. Bei der Parazentese wird Eiter entleert.

Der Zustand am folgenden Tage ziemlich unverändert. Es wurde in Narkose zuerst $L$ u mbalp un ktur vorgenommen, und da bei derselben $20 \mathrm{ccm}$ getrübte Flüssigkeit entleert wird, macht man gleich die TotalanfmeiBelung. Der ostitische ProzeB nur sehr wenig verbreitet, im Antrum Cholesteatommassen. Bei dem Entblößen des Sinus mit der Knochenzange reißt plötzlich die Sinuswand und man bekommt eine heftige Blutung, die doch durch Tamponade beseitigt wird. Die Dura wird entblößt und gespalten, wodurch kein Eiter erscheint, ebensowenig wie bei der folgenden Probeinzision des Hirns.

Der Zustand blieb nach der Operation unverändert, nur trat als neues Symptom noch Nystagmus hinzu. Während der folgenden Nacht Exitus. Mikroskopie der Zerebrospinalflüssigkeit: Ausschließlich polynukleăre Zellen, zahlreiche lanzeolate Diplokokken, die nach Gram färbbar sind.

Bakteriologische Untersuchung: Reinkultur von Pneumococcus Fränkelii lanceolatus.

Auszug aus dem Sektionsprotokoll: Mittlere Temporalwindung (der Kraniotomie entsprechend) ein bischen kontundiert, enthält keinen Abszeß. In der Schädelhöhle etwas seropurulente Flüssigkeit; Pia überall stark eitrig infiltriert; im Eiter Pneumokokken in reichlicher Menge. Schläfenbein: in der Mündung des inneren Gehörgangs ein bischen Eiter. Im Labyrinth kein Eiter, sondern einige Scbwellung und Hyperämie. Dagegen strecken sich einige mit Eiter und Granulationen gefüllte Zellen die hintere Fläche der Pars petrosa entlang bis in die Nähe des Porus acust. int.; keine sichtbare Perforation.

Epikrise: Es handelt sich hier um einen insofern ganz gewöhnlichen Fall als es eine chronische, vernachlässigte Eiterung war, die danu eines Tags eine Meningitis verursachte, die sich nicht durch den operativen Eingriff beeinflussen ließ. Dagegen bot der Fall anfangs einige diagnostische Schwierigkeiten dar. Patientin gehörte einer sehr stark nervös belasteten Familie an und war offenbar selbst in ziemlich hohem Grade hysterisch. In Betracht der nicht besonders ausgesprochenen meningitischen Symptome wußte man deshalb am ersten Tag nicht, ob es sich um eine wirkliche schwere Erkrankung handle oder nur um einen durch ein akutes Auflodern der Ohreiterung hervorgerufenen hysterischen Zustand. Es war ganz unmöglich, eine Lumbalpunktur vorzunehmen, um 
so die Diagnose festzustellen. Da sich während der Nacht der Zustand nicht änderte, mußte man deshalb am folgenden Tag: in Narkose die Lumbalpunktur vornehmen und als diese trübe Flüssigkeit ergab, ließ man gleich die Totalanfmeißelung und Kraniotomie folgen.

3. Anders P., 57 Jahre, aufg. 3. April, gest. 10. April 1907.

Klinische Diagnose: Otits med. supp. chr. s. - Leptomening. purul. diff. - Abscess. cerebri?

Sektionsdiagnose: Leptomening. pur. diff. - Otit. med. supp. s. Symfysis pericardii totalis fibrosa - Endocarditis verrucosa.

Anamnese: Patient hatte als Kind doppelseitige Eiterung, linkes Ohr später gesund, am recbten vor 20 Jahren wieder eine Eiterung. Hat später nichts bemerkt bis er vor 3 Monaten Schmerzen und Ausfluß vom linken Ohr bekam. Er merkt andauernd leichte Schwindelerscheinungen, ferner Gedächtnisschwäche und beschränktes Arbeitsvermögen.

Status praesens: A ur. s.: Eiter nicht übelriechend, große Perforation, Trommelfellrest geschwollen, einige Granulationen. Warze natürlich. - Übrige Untersuchung ergibt nichts Besonderes, auch nicht die Stethoskopie des Herzens. Temp. 38,3 .

5. April. Temp. 37,6-37,2. Total a ufmeiBelung, bei der sowohl Sinus wie Dura in geringer Ausdehnung entblößt werden. Antrum und teilweise Kuppelraum mit Cholesteatom gefüllt, der ostitische Prozeß übrigens nicht sehr verbreitet.

6. April. Temp. 38,2-38,8. Einige Kopfschmerzen und Schwindel. 7. April. Temp. 40,3-40,6. Etwas Übelkeit, kein Erbrechen. "Kernig" vorbanden, übrigens keine meningealen Erscheinungen. 8. April etwas benommen. 9. April unverändert. Augengrund normal. Der Verband wird gewechselt. Im unteren Wundwinkel eine eiternde Fistel, die Haut in der ganzen Ausdehnung der Wunde unterminiert; die Wunde wird deshalb geöffnet und die Tamponade gewechselt; das Aussehen der Kavität sehr gut. - Als nachmittags Nackensteifigkeit auftrat und "Kernig" mehr ausgesprochen war, wurde Lumbalpunktur rorgenommen, "es gelang jedoch nicht auch nur einen einzigen Tropfen Flüssigkeit zu entleeren, obschon man bestimmt das Gefühl hat, als wäre die Kanülenspitze in den Subduralraum hervorgedrungen. - Patient wurde mehr und mehr benommen. Temp. um 40,5 ; am 10. April Exitus.

A uszug a us dem Sektionsprotokoll: Die weichen Hirnhäute eitrig infiltriert, namentlich auf der Konvexität; um beide Nn. acustici herum sowie auf der Oberfläche des Kleinhirns liegt Eiter. In der Schädelhöble einige seropurulente Flüssigkeit. Dura überall glatt, von natürlichem Aussehen, auch an der entblößten Stelle, linkes Tegmen tympani ebenfalls natürlich. Sinus nicht tbrombosiert. Schläfenbein: Es läßt sich kein sichtbarer Infektionsweg auffinden, Schnecke und Bogengänge natürlich, auch findet man keine eiternden Zellen, die sich tiefer in das Felsenbein hinein sich debnen.

Epikrise: Die Art und Weise, in der sich in diesem traurigen Falle die Meningitis entwickelt hat, bleibt unaufgeklārt. Die besproehenen Schwindelerscheinungen sowie das beschränkte Arbeitsvermögen wurden teils als Reizerscheinungen, teils als Zeichen einer leichten Toxämie gedeutet. Auch verlief die Operation in tiblicher Weise; daß die Dura - wie es so oft geschieht - an einer kleinen Stelle entblößt wurde, kann man wohl keine Bedeutung beimessen, namentlich da sich bei der Sektion die Dura an dieser Stelle völlig normal erwies, 
Auch fand man bei der Sektion keine Andentung eines älteren Fokus, der vielleicht dureh die Erschütterung der Meilielschläge hätte aktiv werden können. Man muB also wohl annehmen, daß es sich um eine ohne besondere Veranlassung stattfindende metastatische Infektion gehandelt hat, die nur zufällig mit dem Zeitpunkte der Operation zusammentraf.

4. Arne M., 11 Monate, aufg. 22. Juni, gest. 24. Juni 1907.

Klinische Diagnose: Otitis med. supp. subchr. s. - Leptomeningitis purul.

Sektionsdiagnose: Leptomening. pur. - Otit. med. supp. s. Osteitis oss. temp. - Labyrinthitis supp. - Tuberculosis miliaris.

A namnese: Vor $1 \frac{1}{1}$. Monat Ohrfluß links, vor 3 Wochen Ausfluß spärlicher, gleichzeitig Schmerzen. Vor 4 Tagen wurde das Kind unruhig, klagend, mehrmals Erbrechen, übrigens keine cerebralen Erscheinungen.

status praesens: Ist ziemlich herabgekommen, Temp. 38,6, Puls 150, unregelmäßig. Ausgesprochene Nackensteifigkeit, bedeutender Strabismus convergens, "Kernig“. A u r. s.: Stinkender Eiter, Trommelfell rot, geschwollen, mit einer kleinen Perforation. Geringe Infiltration an der Warze. Bei Lumbalpunktur wird $12 \mathrm{ccm}$ trübe Flüssigkeit entleert, die degenerierte, vermeintlich polynukleäre Zellen und Fränkelsche Diplokokken enthält.

23. Juni. Temp. 38,6-39,2. Sehr unruhig, aufschreiend, fortwăhrendes Schielen; bekommt während der Untersuchung einen Anfall von klonischen Zuckungen, im rechten Arm anfangend und sich dann nach allen Extremitäten verbreitend. Ophthalmoskopie nichts Besonderes. Bei Lumbalpunktur wird $12 \mathrm{ccm}$ noch mehr getrübte Flüssigkeit entleert. Spăter wiederholte Krampfanfälle. 24. Juni. Exitus.

Auszug aus dem Sektionsprotokoll: Diffuse eitrige Meningitis, namentlich auf der Konvexität, auf der Basis nur wenig ausgesprochen. Keine Tuberkeln. Im Eiter findet man Pneumokokken. Miliärtuberkulose der Lungen und mehrerer anderen Organe. Schläfenbein: Über dem Gebörgang, vor und über der Linea temporalis findet man eine, vom Periost bedeckte, pfenniggroße Öffnung im Knochen; man kommt hier in eine Höhle hinein, die mit Eiter und Granulationen sowie mit einzelnen Resten von mürbem Knochengewebe gefüllt ist. Die Höhle steht mit dem Antrum und dem Kuppelraum in offener Verbindung. Von dem besprochenen Fokus heraus strecken sich mit Eiter gefüllte Zellen bis gegen die Spitze des Felsenbeins. Das Labyrinth ist völlig mit schleimigem Eiter gefült.

Epikrise: Es handelt sich hier um eine bei einem mit Miliärtuberkulose behafteten Kinde auftretende otogene Meningitis. Der Zustand erschien schon bei der Aufnabme ein so hoffnungsloser, daß man auch nicht eine Operation versucht hat.

5. Hedvig Dorthea P., ${ }^{1}{ }_{2}$ Jahr, aufg. 10. Ang., gest. 12. Aug. 1907.

Klinische Diagnose: Otitis med. supp. ac. s. - Osteitis proc. mast. s. ac. - Absc. cerebri? - Meningitis?

Sektionsdiagnose: Tubercul. pulm. - Tubercul. miliaris pulm., lienis, hepatis et meningum. - Focus caseosus cerebelli. - Otitis med. pur. s.

A namnese: Früher gesund. Vor 1 Monat beiderseitiger Ohrfluß. Vor 8 Tagen Appetitmangel, bedeutende Trägheit, später heftiges Erbrechen, am Tag vor der Aufnahme völlig soporös, einige Zuckungen in den Extremitäten. Es wurde beiderseits Parazentese vorgenommen.

Status praesens: Aur. d.: Geringe Menge seröser Flüssigkeit, Trommelfell geschwollen. A ur. d.: Stinkender Eiter, Trommelfell rot, geschwollen. Liegt soporös danieder, mit leichten klonischen Zuckungen, Kopf nach links gedreht, wird steif gehalten, Augen deviieren nach links. 
Rechter Arm etwas rigid. Anscheinend leichte Parese des rechten unteren Fazialis. "Kernig“ und "Trousseau" nicht vorbanden, „Babinski" rechts, nicht links. Ophthalmoskopie: Beide Papillen geschwollen, grau verfärbt. Temp. 38,1 , Puls $60-100$.

Bei L umbalpunktur wurde reichliche klare Flüssigkeit entleert, die spärllche mononukleäre Leukozyten enthielt.

Es wird ohne Narkose A ufmeißelung vorgenommen. Der ostitische Prozeß nicht besonders ausgedehnt. Dura der mittleren Schădelgrube wird entblößt und gespalten, das Hirngewebe prolabiert etwas; Probeinzision nach verschiedenen Richtungen fordert keinen Eiter zutage; dann werden Sinus und Dura der hinteren Schādelgrube entblößt, Punktur des Kleinhirns ebenfalls negativ. Exzidiertes Hirngewebe zeigt mikroskopisch nichts Krankhaftes.

Nach der Operation war der Puls etwas schneller und regelmäßiger, Zustand übrigens unverändert. Stethosk opisch werden zahlreiche feine Rasselgeräusche gefunden, keine Dämpfung. Am 12. Juli. Exitus.

Auszug a u dem Sektions protokoll: Die Cerebrospinalflussigkeit nicht besonders reichlich, Meningen nicht injiziert. Um den Stiel des Kleinhirns berum sieht man in den Hirnhäuten stecknadelkopfgroße gelbe eitrige Infiltrate ferner eine Anzahl sehr kleiner miliären Tuberkeln, die sich in weiterer Ausdebnung an der Hirnbasis verfolgen lassen. Im linken vorderen oberen Kleinhirnlappen ein erbsengroßer kaseöser Fokus, der die Oberfläche erreicht. Die Hirnventrikeln etwas dilatiert. Die Konsistenz und Blutfüllung des Hirngewebes natürlich. Mikroskopie des Hirngewebes ergab auch nichts Pathologisches.

Epikrise: Wir haben es hier mit einem Fall zu tun, in dem man vornherein einen HirnabzeB erwarten mußte, indem verschiedene Lokalsymptome, Drehung des Kopfes, konjugierte Deviation der Augen, Fazialislähmung, vorhanden waren, dagegen keine meningitischen Erscheinungen. Tatsächlich war aber kein Abszeß vorhanden und das Leiden war eine Miliartuberkulose, die mit der Ohrenkrankheit des Kindes in keinem Zusammenhang stand.

6. Valdemar August M., 51 Jahre, aufg. 26. Juli 1907, gest. 27. Juli 1907.

Klinische Diagnose: Otits med. supp. ac. s. - Osteitis proc. mast. s. ac. - Periphlebitis sin. transv. - Leptomeningitis diff. purul. Abscessus cerebri?

Sektionsdiagnose: Meningitis purul. diff. - Otit. med. pur. s.

An a mnese: Seit 5 Monaten Ohreiterung links; es wurde damals zweimal von einem Spezialisten Parazentese vorgenommen, später hat sich Patient jeder ärztlichen Behandlung entzogen. Fast während der ganzen Zeit haben Schmerzen bestanden, sowie auch Schwellung hinter dem Ohre; beträchtliche Kopfschmerzen, sodaß er sich nicht beugen konnte. Während der letzten paar Monate nachts immer etwas Fieber, während er sich am Tag leidlich wohl befand; zuletzt fing er an abends benommen und soporös zu werden.

Status praesens: Patient ist soporös, reagiert nur auf sehr energische Ansprache, vermag doch nicht zu antworten, străubt sich gegen Untersuchung. Leichte rechtsseitige Fazialisläbmung. Nackensteifigkeit und „Kernig“ vorhanden. A u r. s.: Stinkender Eiter. Warzenfortsatz geschwollen. Lumbalpunktur wird versucht, gelingt aber nicht.

Es wird sofort A ufmeißelung vorgenommen; man findet einen ziemlich verbreiteten ostitischen Prozeß, der an einer Stelle auch die Dura erreicht hat, sodaß diese mit Granulationen bekleidet ist. Dura wird in weiter Ausdehnung entblößt und gespalten; demnächst wird im Hirn Probeinzision vorgenommen, jedoch kein Eiter gefunden. Auch Sinus wird entblößt, der 
entzundliche Prozeß hat auch ihn erreicht, sodaß man direkt auf der Sinuswand zerfallende Granulationen findet.

Zustand nach der Operation im wesentlichen unverändert, bis Patient am folgenden Morgen zu kollabieren anfing; während des Vormittags Exitus.

A uszug aus dem Sektionsprotokoll: Die Wand des Sinus transversus fibrös verdickt, kein Thrombus. Das Hirngewebe ist der Kraniotomieöffinung entsprechend etwas nekrotisiert, übrigens normal. Die weichen Hirnhănte überall ödematös geschwollen und bis in die Tiefe aller Furchen eitrig infiltriert. Reichliche seropurulente Flüssigkeit in der Schädelböhle.

Epikrise: Auch in diesem Falle hatte man neben der recht unzweifelbaften Meningitis einen Hirnabszeb erwartet; ein solcher war aber nieht vorhanden und das langdauernde Unwohlsein mit cerebralen Erscbeinungen mag dann auf einer Toxämie beruht haben. Übrigens bietet der Fall kein besonderes Interesse dar.

7. Eleonora Christine H., 11 Jahre, aufg. 9. September 1907, gest. 20. September 1907.

Klin is che Diagnose: Otit. med. supp. chr. d. - Ostit. proc, mast. d. chr. - Meningitis tuberc. - Bronchitis.

Sektionsdiagnose: Meningitis tuberc. - Tubercul. miliar. - Foci tub. vet. pulm.

Anamnese: Man bekommt über den bisherigen Verlauf der Krankheit nur sehr spärliche Ausküntte; Patientin weib nur, dab sie einige Zeit Koptweh gehabt, ferner ein paarmal Erbrechen, das vielleicht durch den Genuß unreifen Obstes verursacht wurde. Obrenfluß soll seit langer Zeit nicht bestanden haben.

Status praesens: Patientin ist mager und bleich, völlig klar, aber sehr träge. Keine Nackensteifigkeit Kernig recht ausgesprochen. Ist beim Berühren der Beine sehr emptindlich. Aur. d.: Geringe Menge stinkenden Eiters, Boden durch Detritus verdeckt. Leichte Druckempfindlichkeit über der Fovea mast. Temp. 38,1, Puls 88, regelmäbig.

10. Sept. Zustand unverändert. Bedeutende kutane Hyperästhesie. Es wird $L u m b a l p u n k t u r$ vorgenommen mit Entleerung einer nicht absolnt klaren, mit zahlreichen feinen weißen Schuppen versehenen Flüssigkeit. Demnäcbst wird Totalaufmeißelung vorgenommen. Es wird ein ziemlich verbreiteter ostitiscber Prozeß anfgetunden, der an ein paar Stellen die Dura erreicht; diese ist etwas verdickt und rötlieb. Mikroskopie der Cerebrospinalfüssigkeit ergibt eine zitmliche Menge von Leukozyten, ausschließlich mononukleäre; keine Mikroben.

Der Zustand hielt sich unverăndert. Ophthalmoskopie normal. 14. Sept. Temp. 39, soporös, bisweilen benommen. Während der folgenden Tage nahmen Sopor und Benommenbeit za und am 20. Sept. trat Exitus ein.

Epikrise: Daß es sich hier um eine Meningitis handelte war von vornherein klar, ob aber eine otogene oder eine tuberkulöse konnte erst bei der mikroskopischen Untersuchung: der Cerebrospinalflüssigkeit erörtert werden; da aber doch Zeichen einer Mastoiditis vorhanden waren und die Meningitis, falls sie otogenen Ursprungs war, doch wohl kaum noeb besonders hervorgeschritten war, machte man gleich die TotalaufmeiBelung, was ja aber für den weiteren Verlauf der Krankheit ganz belanglos blieb.

Archiv f. Ohrenheilkunde. Bd. 77. 
8. Sophie Magdalene H., 52 Jahre, aufg. 19. August 1907, gest. 1. Oktober 1907.

Klinische Diagnose: Otit. med. supp. ac. d. - Ostit. proc. mast. d. ac. - Pyaemia otogenica - Infarctus pulm. sin.

Sektion nicht erlaubt.

Anamnese: Vor 5 Tagen Ohrsausen rechts, später auch Schmerzen und Schwerhörigkeit. Kein Schwindel oder Erbrechen, dagegen Kopfschmerzen, namentlich in der rechten Kopfhälfte.

Status praesens: Aur. d.: Im Gehörgang eine geringe Menge Flüssigkeit, Trommelfell rot, geschwollen, keine deutliche Perforation. An der Warze einige diffuse Schwellung und Empfindlichkeit. Es wird Parazentese vorgenommen.

Wăhrend der folgenden Tage befand sich Patientin ganz gut, Temp. nur sebr wenig erhōbt. Da aber nach 10-11 Tagen das Trommelfell fortwăhrend ziemlich geschwollen war, die Temp. noch immer mäßig erhōht und Patientin außerdem tber Druckgeftihl im Kopfe klagte, wurde am 31. August A u fmeiBelung vorgenommen. Der Warzenfortsatz ist in seiner ganzen Ausdehnung der Sitz einer recht bedeutenden akuten Ostitis; in der Tiefe der Spitze ist das Knochengewebe stark brüchig und bei der Auslöffelung kommt hier aus dem unteren horizontalen Schenkel des Sinus eine nicht beträchtliche Blutung. Bakteriologische Untersuchung des Eiters: Staphylococcus pyogenes albus.

1. Sept. 'Temp. 37,7-40. Befand sich recht wohl nach der Operation; um Mitternacht ein Schüttelfrost. 2. Sept Temp. 40,1-38,6. Wohlbefinden. 3. Sept. Temp. 39-38,6. Wiederum ein Schüttelfrost. Klagt über Stechen in der linken Seite, etwa in der Gegend des lctus; 1 Herzton etwas dumpf und ausgezogen. 4. Sept. Etwas Husten, der Auswurf zähe, rotfarbig; in der linken Infrascapularis leichte Dämpfung und bronchöse Respiration. Während der folgenden Tage hielt sich der Zustand ziemlich unverändert. Temp. meist 38-40, durchgehends einmal täglich ein Schüttelfrost, die Kräfte ganz gut. $0 \mathrm{ph}$ thalmoskopie gibt nichts Besonderes. Am 10. Sept. wurde der Verband zum erstenmal gewechselt. Die Wundhöhle durchgehends ganz gut; beim Entfernen des auf den Sinus gedrückten Gazestreifens tritt profuse Blutung anf, sodaß sofort tamponiert werden muß. - Zustand noch immer unverändert, nur der Husten geringer; stethoskopisch werden jetzt nur zerstreute feuchte Rasselgerăusche gefunden. Am 13. Sept. ein Schüttelfrost, dér etwa $3 / 4$ Stunde dauerte und von Cyanose begleitet war, ebenso am 14., am 15. und 16. dagegen Woblbefinden. Am 17. wird der Verband wiederum gewechselt, diesmal keine Blutung, die Wundhöhle frisch granulierend. Etwa vom 20. an fingen die Schüttelfröste wieder an und jetzt trat auch allmählich Krăfteverfall ein, vom 26 . an wurde sie mehr und mehr soporös, bis schließlich ohne neue Symptome am 1. Okt. Exitus eintrat. Die Temperaturkurve zeigte während des ganzen letzten Teils des Krankheitsverlaufs einen ausgesprochenen pyämischen Typus mit ziemlich großen Remissionen.

Sektion wurde nicht erlaubt, es wurde aber nach dem Tode die Wunde so viel erweitert, daß man den restierenden Teil des Warzenfortsatzes resezieren und Sinus transversus und Bulbus jugularis in toto entfernen konnte. Die innere Wand dieser Gefäße schien völlig gesund und im Hoblraum lag ein ganz frisches Gerinnsel; an dem bei der Operation entblößten Teil des Sinus fand man an der Außenwand einige Bindegewebsbildung. Beim Entfernen des vorderen Teils des Warzenfortsatzes kommt ein einzelner Tropfen Eiter hervor, obne daß es gelingt zu entdecken, woher er kommt; wabrscheinlich stammt er aus irgend einer kleinen bei der Operation nicht aufgefundenen Zelle.

Mikroskopische Untersuchung des entfernten Sinus und Bul. bus: Keine Entzündungserscheinungen oder andere pathologische Veränderungen.

Epikrise: Es schien in diesem Falle anfangs sich um eine ganz akute Mittelohreiterung zu handeln, die ohendrein recht 
frth zur Behandlung kommt. Nach 10-11 Tagen bestehen aber noch Schmerzen und Fieber, kurz Retentionserscheinungen; es wird deshalb Resektion gemacht. Da tritt in der folgenden Nacht ein Schüttelfrost auf und es entwickelt sich das Bild einer typischen otogenen Pyämie mit springenden Temperaturen, Schüttelfrösten und Lungenembolie, vielleicht auch einer Endokarditis. Die Kräfte halten sich lange Zeit hindurch erstaunlich gut, da aber die Pyämie noch immer fortdauert, reichen sie schließlich nicht mehr, Patientin wird soporös und nach einigen Tagen tritt Exitus ein. Im ersten Augenblick konnte man daran denken, daß die akzidentelle Sinusläsion bei der Operation mit dem Entstehen der Pyämie in Verbindung stände; sie wäre dann aber wohl kaum so schnell nach der Operation aufgetreten und auch mußte sie durch eine Sinusthrombose vermittelt sein; eine solche war ja aber nicht vorhanden und die Läsionsstelle war tadellos geheilt. Leider konnte keine vollständige Sektion vorgenommen werden, sodaß es unaufgeklärt bleibt, in welcher Weise sich die Infektion vom Mittelohr aus fortgepflanzt hat, jedenfalls also nicht durch eine Thrombose - auch nicht wandständige - des Sinus transversus.

Der folgende Fall soll auch hier mitgenommen werden, indem der Patient, obschon nicht in der Klinik gestorben, doch lange Zeit hier behandelt wurde.

9. Sören Niels S., 37 Jahre, aufg. 10. April 1907, in d. Neuro]. Abt. 6. Mai 1907 (gest. 12. Mai 1907).

Klinische Diagnose; Otits med, supp. chr. s. - Abscessus cerebri. - Encephalitis progressiva.

Sektionsdiagnose:

A namnese: Seit der Kindheit ohrenkrank, in 1891 Totalaufmeißelung rechts. Einige Zeit Ohrenfluß links; fing vor ein paar Tagen an nachts unruhig und benommen $z u$ sein. Gestern einige Nackensteifigkeit, "Kernig“" vorhanden, etwas somnolent, Ophthalmoskopie: mittelstarke Stauungspapille.

Status praesens: Befindet sich heute bedeutend besser, nur ein bischen Kopfweh; keine Benommenheit, beantwortet Fragen ohne Zögern; das Gedächtnis scheint dagegen etwas geschwächt zu sein; keine Aphasie oder Paraphasie. Temp. 39,6 , Puls 88 , regelmäßig kräftig.

11. April wird Lumbalpunktur vorgenommen mit Entleerung von $40 \mathrm{ccm}$ leicht unklarer Flüssigkeit, die unter erhöhtem Druck ausströmt. Gleich darauf Totalaufmeißelung, bei der man die Zellen in der Tiefe des Warzenfortsatzes mit Granulationen gefüllt tindet. Sinus und Dura werden entblößt, beide erscheinen normal; Dura der mittleren Schädelgrube wird gespalten und das Gehirn inzidiert; in der Richtung nach innen vorn findet man Eiter und bei der Dilatation fließt eine ziemlich große Menge ichorösen, mit nekrotischen Gewebsfetzen vermischten Eiters ab. Es wird ein Drain eingelegt.

12. April. Befindet sich nacb der Operation sehr wohl, ist sehr froh und zufrieden. Temp. 38,7-37,8. Ophthalmoskopie (unnittelbar nach der Operation vorgenommen) ergab natürliche Verhältnisse. Keine Nackensteifigkeit, "Kernig" angedeutet. Befand sich auch während der folgenden 
Tage völlig wohl, Temp. fast normal, nur die Sprache war etwas langsam. 15. April. Temp. 39,8-38,2. Während der Nacht etwas unruhig, befindet sich heute morgen wohl. - Während des Tags war jedoch Patient etwas träge und apathisch und bei der Abendvisite fand man ausgesprochene Paraphasie. Er benennt vorgezeigte Gegenstande falsch, scheint sich doch oftmals bewuBt zu sein, daB es falsch ist, namentlich nennt er alles Mögliche für "Nadel" (Patient ist Schneider); beim Schreiben sind die Buchstaben gut geformt, er schreibt aber dieselben falschen Benennungen; beim Nachsprechen dagegen wiederholt er selbst ziemlich schwere Worte richtig. Seinen Namen und seine Wohnung schreibt er richtig. Das Schreiben nach Diktat fält ihm schwer und er schreibt oft falsche Buchstaben. Das Leseu geht sehr gut vonstatten, ebenso das Abschreiben nach einem Buch. Bemerkenswert ist, daß or sämtliche Geldstäcke kennt und richtig benennt. Am 16. wird die Wunde revidiert; aus der Abszeßhöhle tließen einige mit Eiter gemischte nekrotische Fetzen. Bei Lumbalpunktur werden $25 \mathrm{ccm}$ klarer Fläsigkeit entleert. - Nachdem sich jetzt der Zustand einige Tage hindurch fast unverändert gehalten hatte bemerkte man erst eine zunehmende Schwerhörigkeit, dann Sopor und mehr und mehr ausgesprochene Aphasie, sodaß er bisweilen nur ganz sinnlose Lautverbindungen hervorzufuhren vermag; er versteht dagegen alles. Die Temperatur, die zur Zeit der oben besprochenen Revision erhöht war, hielt sich jetzt normal; der aus der Abszeßnöhle kommende Eiter, der etwas übelriechend gewesen, ist jetzt nach der Verwendung von Wasserstoffsuperoxyd und Jodoformgaze von gutem Aussehen und ohne üblen Geruch. Eine in die Fistel hineingeführte Sonde gleitet ohne dem geringsten Widerstand $2 \mathrm{a}$ begegnen $9 \mathrm{~cm}$ in die Tiefe in der Richtung nach innen oben und ein wenig nach vorn. Am 21. wird eine neue Kraniotomie vorgenommen, indem etwa $2 \mathrm{cr}$ über dem Rande der früheren Offrnung eine neue Óffnung im $O_{s}$ parietis anlegt; Dura wird hier gespalten und das Gehirm nach verschiedenen Richtungen hin inzidiert ohne daß Eiter zum Vorschein kommt. 25. fand man eine recht ausgesprochene Parese der rechtsseitigen Extremitäten, dagegen keine Fazialis-noch Augenmuskel-Läbmungen. Augengrund fortwăbrend natürlich.

Wabrend der folgenden Zeit war der Zustand etwas wechselnd, die Parese der Extremităten nabm vorläufig etwas ab, um später wieder zazunehmen, dagegen fand sich eine Fazialisparese ein; fast vollständige Aphasie, dagegen scheint er alles zu verstehen. Temperatur nur wenig erhöht. Aus beiden Kraniotomieöffnungen wuchert das Hirngewebe hervor, in der unteren ist es anßerdem stark nekrotisch und übelriechend.

Weil der Geruch den andern Patienten sehr lästig ist und seine Unruhe sie im Schlafen stört, wird Patient, da er in der Klinik nicht isoliert werden kann, in die neurologische Abteilung übersiedelt. Der Zustand blieb hier ziemlich unverändert bis am 12. Mai Exitus eintrat.

Auszug aus dem Sektionsprotokoll: Durch die Trepanationsöffnung dringt eine Sonde in eine große Abszeßhöhle hinein; nach dem Entfernen des oberen Teils des Schädels ergibt sich, daß der Abszeß fast den ganzen linken Temporallappen destruiert hat und ferner in den Ventrikel himein gebrochen ist. Der Eiter ist hochgradig übelriechend. Keine Meningitis ausgenommen die nächsten Umgebungen der Trepanationsöffnung.

Mikroskopie der Cerebrospinalfussigkeit: 11. April. Zablreiche polynukleäre Leukozyten und Endothelien; keine Mikroorganismen; später 16.: Spärliche Zellen, teils Leuko-, teils Lymphozyten, ferner einige Lndothelien. Bakteriologische Untersuchung: Eiter ans dem Warzenfortsatz: Reinkultur von kleinen weißen Kolonien eines pneumokokkenäbnlichen Diplokokken. Eiter aus dem HirnabszeB: Teils Kolonien desselben Diplokokkus, wie oben, teils diffuser Anwachs eines beweglichen coli-ähnlichen Stäbchens.

Epikrise: Es handelt sieh hier um einen Fall von ehro. nischer Eiterung, in dem ziemlich plötzlich cerebrale Erseheinungen eintreten und zwar lassen diese am ehesten eine otogene 
Meningitis vermuten, es war Fieber vorhanden, Benommenheit, anfangs auch leichte Nackensteifigkeit und Kernig; ferner wurde bei der Lumbalpunktur eine große Menge schwach getrübter Flüssigkeit entleert, die auch mikroskopisch polynukleäre Leukozyten enthielt; von fokalen Erscheinungen waren dagegen keine vorhanden. Trotzdem wurde bei der Operation ein ziemlich großer Hirnabszeß entdeckt und nach dessen Entleerung schien alles gut zu gehen, nur machte sich einige "slow cerebration" bemerkbar. Dann treten 4 Tage nach der Operation die ersten fokalen Erscheinungen auf in Gestalt einer sich vorläufig wesentlich nur als Paraphasie äußernden motorische Aphasie. Trotz der sorgfältigsten Behandlung breitet sich offenbar die Entzündung immer weiter aus, die Höhle wird größer und größer und das umgebende Hirngewebe wuchert erst aus der Kraniotomieöffnung heraus, zerfällt dann in Nekrose; durch eine neue Kraniotomie denkt man vielleicht einen andern Abszeß aufzudecken oder auch bessere Abflußbedingungen für den Eiter zu schaffen; es hilft aber nicht. Die Sprachstörung entwickelt sich jetzt nach und nach zu einer vollständigen motorischen Aphasie, während die sensorische Sphäre völlig unbeeinträchtigt scheint. 10 Tage nach dem Auftreten der ersten aphatischen Erscheinungen kommt dann eine Parese der rechtsseitigen Extremitäten hinzu, später auch noch eine Fazialislähmung. Die Lähmungen wechselten sehr in Intensität; bisweilen konnte er die Extremitäten ganz gut bewegen, dann wieder wurden sie völlig schlaff und unbeweglich. Bei der Sektion fand man den Schläfenlappen fast völlig zerstört, während in der Gegend der motorischen Zentren sowie des Brocaschen Zentrums keine pathologischen Veränderungen aufzufinden waren. Der Schläfenlappenabszeß hat also hier nicht wie gewöhnlich als Folge der Zerstörung des Wernickeschen Zentrums eine sensorische Aphasie verursacht sondern vielmehr eine ganz typische motorische Aphasie und zwar in Verbindung mit Leiden anderer motorischer Zentren; es müssen demnach die betreffenden Erscheinungen als anf indirektem Wege, durch den Druck, entstanden erklärt werden, womit auch der starke Wechsel in der Intensität der verschiedenen Erscheinungen übereinstimmt.

Von nicht letal verlaufenden endokraniellen Komplikationen wurden folgende 3 Fälle behandelt:

1. Svend Aage F., 8 Jahre, aufg. 7. Aug. 1907, entl. 8. Sept. 1907

Klinische Diagnose: Otitis med. supp. ehr. s. - Ostitis proc. mast. s. chr. - Abscessus perisinuosus. - Phlebitis sin. transversi sin. Thrombosis sin. transv. sin. 
A namnese: Seit vielen Jahren Ohrfluß links. Vor 14 Tagen erkrankt, mit Schwindelerscheinungen und Kopfweh, seit dem 4. Tag täglich Erbrechen; war anfangs etwas benommen, später nicht; Kopfschmerzen wesentlich zum Hinterkopf lokalisiert, Nackensteifigkeit soll auch beobachtet sein; hat laut aufgesehrien; keine Krämpfe. Während der letzten Tage ein paarmal Schüttelfrost. Temp. gestern $40,3-40,2$.

Status praesens: Liegt still danieder, etwas soporös, klagt bisweilen. Wird durch Ansprache leicht erweckt und beantwortet mit gewöhnlicher Konversationsstimme hervorgeführte Fragen vernünftig. Liegt mit aufgezogenen Extremitäten; beim Sitzen etwas Schwindelgefübl. Ausgesprochene Nackensteifigkeit; der Kopf wird in nach hinten gebogener Stellung gebalten, dagegen keine Rückwärtsbeugung des Rückens. "Kernig" und ,Trousseau" ausgesprochen, ,Babinski" beiderseits vorhanden. Ophthalmoskopie natürlich. A ur. s. Stinkender Eiter, großer zentraler Trommelfelldefekt; am unteren Teil des Trommelfellrests kleine Granulationen. Die Labyrinthwand geschwollen und rot, leicht blutend. Keine Schwellung des Warzenfortsatzes. Temp. 37,5, Puls 56, leicht unregelmäßig, Resp, 22. Bei Lumbalpunktur wird $10 \mathrm{ccm}$ völlig wasserklare Flüssigkeit entleert die bei Zentrifugierung keinen Niederschlag gibt und auch bei der Mikroskopie keine Formelelemente zeigt.

Es wird sogleich TotalaufmeiBelung vorgenommen. Der ostitische Prozeß ziemlich beschränkt, Tegmen antri in $1 / 2 \mathrm{~cm}$ Ausdehnung destruiert, Dura jedoch hier von natürlichem Aussehen. Nach hinten ein kleiner direkt an die Sinuswand reichender, eitergefüllter Hohlraum; die Sinuswand selbst verdickt. Bei Punktur des Sinus kein Blut, er wird deshalb gespalten, enthält einen roten, nicht zerfallenden Thrombus, der ausgelöffelt wird, bis aus dem oberen Ende Blutung kommt; nach unten erreicht man nicht das Ende des Thrombus; die äußere Sinuswand wird exstirpiert und dann alles tamponiert.

Der Zustand nach der Operation schien entschieden besser, doch war er noch einige Tage hindurch etwas soporös und die Temperatur war leicht erhöht. Während sich die Nackensteifigkeit schnell verlor, konnte am 13. noch Trousseau nachgewiesen werden und noch am 22. bestand Andeutuug von Kernig. Ein paarmal traten noch Temperatur-Erhöhungen auf, einmal sogar bis auf 40,4 ohne das der Verlauf sonst beeinflußt war. Am 1. Sept. konnte er das Bett verlassen und am 8. wurde er zur poliklinischen Nachbehandlung entlassen.

Die Kraniotomiewunde ist jetzt schon längst geheilt, während die Kavität im Mittelohr noch immer eitert.

Epikrise: Es bestanden in diesem Falle so ausgesprochene meningitisehe Erscheinungen, daß man selbst nachdem die Lumbalpunktur keine Trübung des Liquor gegeben hatte doch am meisten geneigt war diese Diagnose festzuhalten; es könnte ja sein, daß die Meningitis |noch verbältnismäßig beschränkt war und den Spinalkanal nicht ergriffen hatte. Daß dem aber nichtso war, beweist der spätereVerlauf, indem nach Eröffnen des perisinuösen Abszesses und Ausräumung des Thrombus die meningealen Symptome allmählich nachließen. Der Thrombus kann es wohl kaum sein, der die Erscheinungen veranlaßt hat, indem er von ganz frischem Aussehen war, nicht zerfallen, auch zeigte eine bakteriologisehe Untersuchung, daß er steril war; es muß also der perisinuöse Abszel gewesen sein und man ersieht hieraus wie große Bedeutung ein soleher Fokus haben kann, selbst wenn er, wie in diesem Fall, ganz klein ist. 
2. Kathinka N., 11 Jahre, aufg. 2. Sept. 1907.

Klinische Diagnose: Otitis med. supp. ac. s. - Ostitis proc. mast. s. ac. - Abscessus perisinuosus. - Thrombophlebitis sin. transv. - Myxoedema. - Lues congenita.

Anamnese: Patientin, die in hohem Grade an Myxoedem leidet, wurde am 11. Juni wegen lmpetigo und Scrophulosis in die dermatologische $A b$ teilung aufgenommen. Am 28. Aug. Temp. 40,2. Kopfweh und Übelsein, Röte und Schwellung hinter dem linken Ohre sowie vom Ohre selbst, am 30. Druckempfindlichkeit, am 31. stinkender Ohrfluß. Da am 2. Sept. der Zustand ziemlich unverändert ist und die Temp. 40,2-38,7 wird Patientin in die Ohrenklinik übersiedelt. Sie hat nicht über Schmerzen geklagt (wobei doch zu bemerken ist, daß sie sehr stupid und träge ist); Hirnsymptome nicht beobachtet.

Status praesens: Nur wenig Eiter, etwas übelriechend, Trommelfell rot, geschwollen, mit einer kleinen Perforation nach hinten unten. $\mathrm{Pa}$ stöse Schwellung und Druckempfindlichkeit über der Warze.

$\mathrm{Am}$ 3. Sept. wurde Resectio proc. mast. vorgenommen; der Warzenfortsatz fast in seiner ganzen Ausdehnung ostitisch verăndert; ein kleiner Fistelgang führt in eine an der Medialseite der Spitze liegende kleine Abszeßhöhle hinein. Der Sinus wird in einiger Ausdehnung entblößt. er ist teilweise von Eiter umgeben und seine Wand ist verdickt, er pulsiert inzwischen und scheint auch sich zu expandieren, sodaß man annehmen darf, daß er flüssiges Blut enthält, Da die Temperatur nach der Operation fortwährend erhổht ist und der ganze Zustand sich nicht wesentlich gebessert hat, wird am 6. Kraniotomie vorgenommen und der Sinus punktiert, wobei Eiter entleert wird; er wird deshalb in weiter Ausdehnung entblößt und seine laterale Wand entfernt; nur der mittlere Teil des senkrechten Schenkels enthält Eiter, während nach oben und unten die Thrombenmassen rötlich und von frischem Aussehen sind. Nach unten zu kommuniziert der Eiter durch eine Perforation der Sinuswand mit dem perisinuösen Abszeß. Der Thrombus wird ausgelöffelt, doch gelingt es nicht, sein distales Ende zu erreichen, indem $4 \mathrm{~cm}$ vom oberen Knie ein zwar dünner, aber sehr kräftiger Blutstrom herausstürzt, der von einer stricknadelgroßen Öffnung in der medialen Sinuswand herrührt; die Blutung läßt sich nicht soweit bewältigen, daß man weiter vorzudringen vermag.

Der Zustand hielt sich auch nach dieser zweiten Operation eine Zeitlang unverändert, die Temperatur war fortwährend erhöht, zeigte teilweise pyämischen Typus; etwa vom 22, an wurde sie aber entschieden besser und konnte am 29. Sept. das Bett verlassen. Die Eiterung aus dem Gehörgang hatte damals schon anfgehört, während die Heilung der Wunde nur sehr langsamen Fortschritt machte. Zur Zeit ist die Wunde zwar ganz oberflächlich, die Granulationen jedoch sehr schlaff und schlecht, mit schmierigem Belag Patientin verweilt noch in der Klinik, ihr Allgemeinzustand hat sich bei Thyreoidinbehandlung bedeutend gebessert, das ganze Aussehen ist liesser und sie ist bei weitem nicht so stupid. Sie wird in der nächsten Zeit an ein Sanatorium zur weiteren Behandlung ihres Myxoedems und ihrer Skrophulose übergehen.

Epikrise: Während in dem vorigen Fall ein ganz kleiner perisinuöser Abszeß ganz bedeutende cerebrale Erscheinungen hervorrief, findet man hier umgekehrt einen ziemlich ausgedehnten perisinuösen Abszeß und obendrein eine mit diesem direkt kommunizierende eitrige Sinusthrombose, die außer der Temperatursteigerung und einigem allgemeinen Unwohlsein keine besonderen Symptome gibt. Der spätere Verlauf war insofern befriedigend als sie sich jetzt sehr wohl befindet, der Zustand der Wunde aber ist ein sehr schlechter, was doch nicht 
Wunder nehmen darf, wenn man sich erinnert, dab drei so bedeutungsvolle konstitutionelle Krankheiten wie Myxoedem, Skrophulose und hereditäre Syphilis dazu beitragen die ganze Vitalität herabzusetzen.

3. Cornelius Georg J., 11 Jabre, aufg. 11. September 1907, ent. 7. Okt. 1907.

Klinische Diagnose: Otitis med. supp. chr. d. cholest. - Ostitis proc. mast. d. chr. - Abscessus subperiostalis reg. mast. - Abscessus perisinnosus.

Anamese: Von dem Patienten folgen keine Auskünte; selber vermag er nur mitzutellon, dals das rechte ohr jeden sommer zu lauten pflegt und daB er jetzt seit 3 Tagen Schmerzen und Schwellung hinter dem Ohre hat.

Status praesens: Aur, d.: Reichlicher stinkender Eiter, Granulationen nach unten vorn, nach oben eine nach dem Antrum führende Fistel. - Am Warzenfortsatz bedeutende, fluktuierende, sehr empfindliche Schwellang, Temp. $38, \bar{s}$.

\section{Tabelle über die im Jahre 1907}

I. Totalanf -

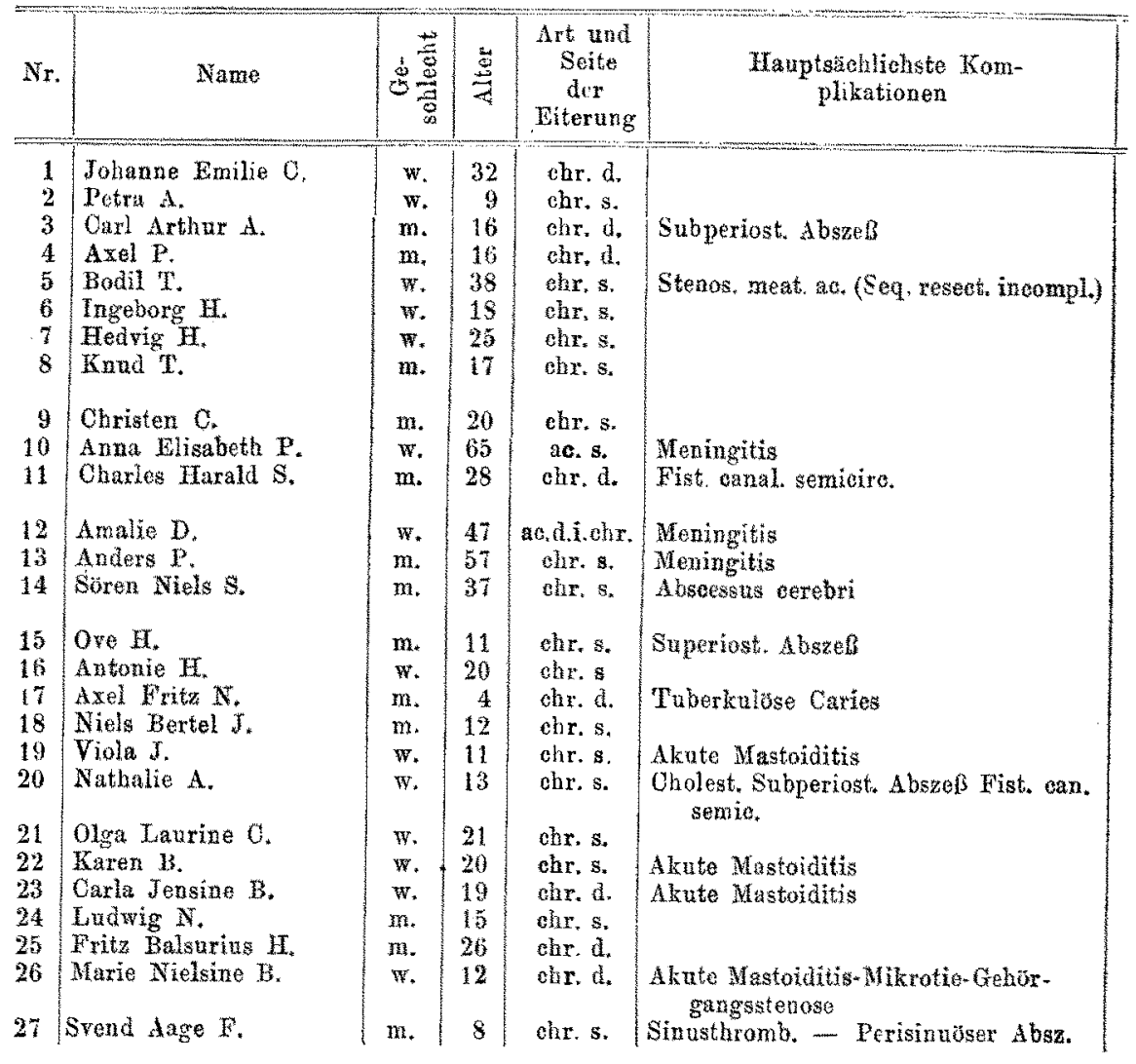


Am 1. Sept. wird Totala ufmeißelung vorgenommen. Aus einem subperiostalen Abszeß wird etwa $10 \mathrm{cbm}$ Eiter entleert; hinter der Gebörgangsöff̈nung eine kleine Fistelöffnung. Im Antrum Cholesteatommassen. Von Antrum aus führt eine Fistel nach hinten und indem man diese weiter verfolgt, stromt etwas Eiter herans; bei dem Entblößen des Sinus wird dann etwa $10 \mathrm{ccm}$ Eiter aus dem Epiduralraum entleert; die Sinuswand an dieser Stelle mit Granulationen belleidet; Sinus und Dura uberhanpt etwas verdickt, aber ubrigens gesund.

Ưber den späteren Verlauf ist eigentlich nicht viel zu berichten, Pailent blieb fieberfrei und befand sich uberhaupt sehr wohl, die große Wunde vernarbte allmählich, die Hohle ist aber noch nicht völlig epidermisiert.

Epikrise: Auch in diesem Falle haben wir es mit einem perisinuösen $A b s z e B$ zu tun, der aber tberbaupt keine Symptome gibt, nicht einmal etwas Fieber; ware nicht die Sohwellung hinter dem Ohre gewesen, wäre Patient wohl tuberhaupt nicht zur Behandlung: gekommen, bis einmal ernstere Erscheinungen angetreten wären und es vielleicht zu spät war, einen Eingriff vorzunehmen.

\section{vorgenommenen Mastoidoperationen.}

m e i $B$ elu $g$ g $n$.

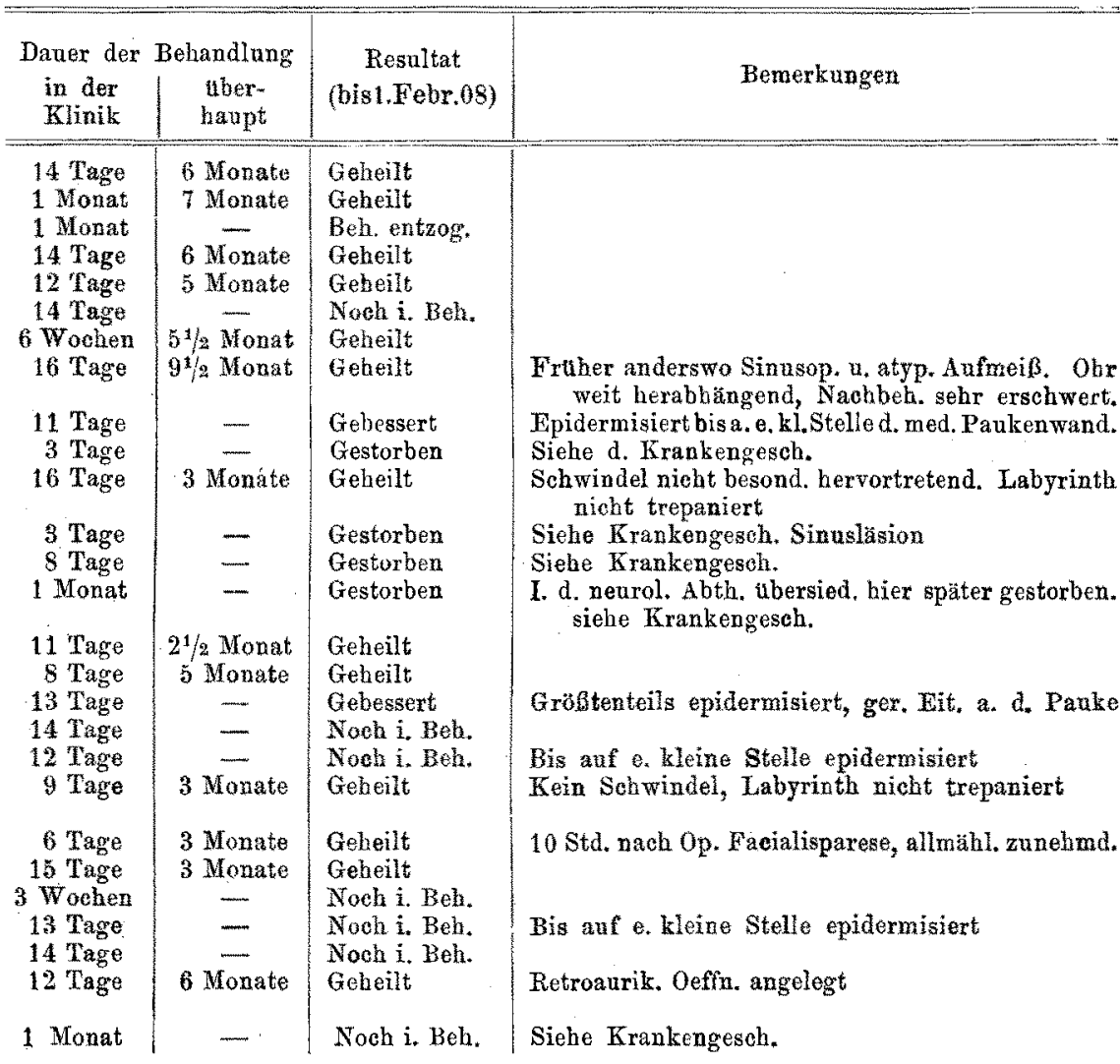




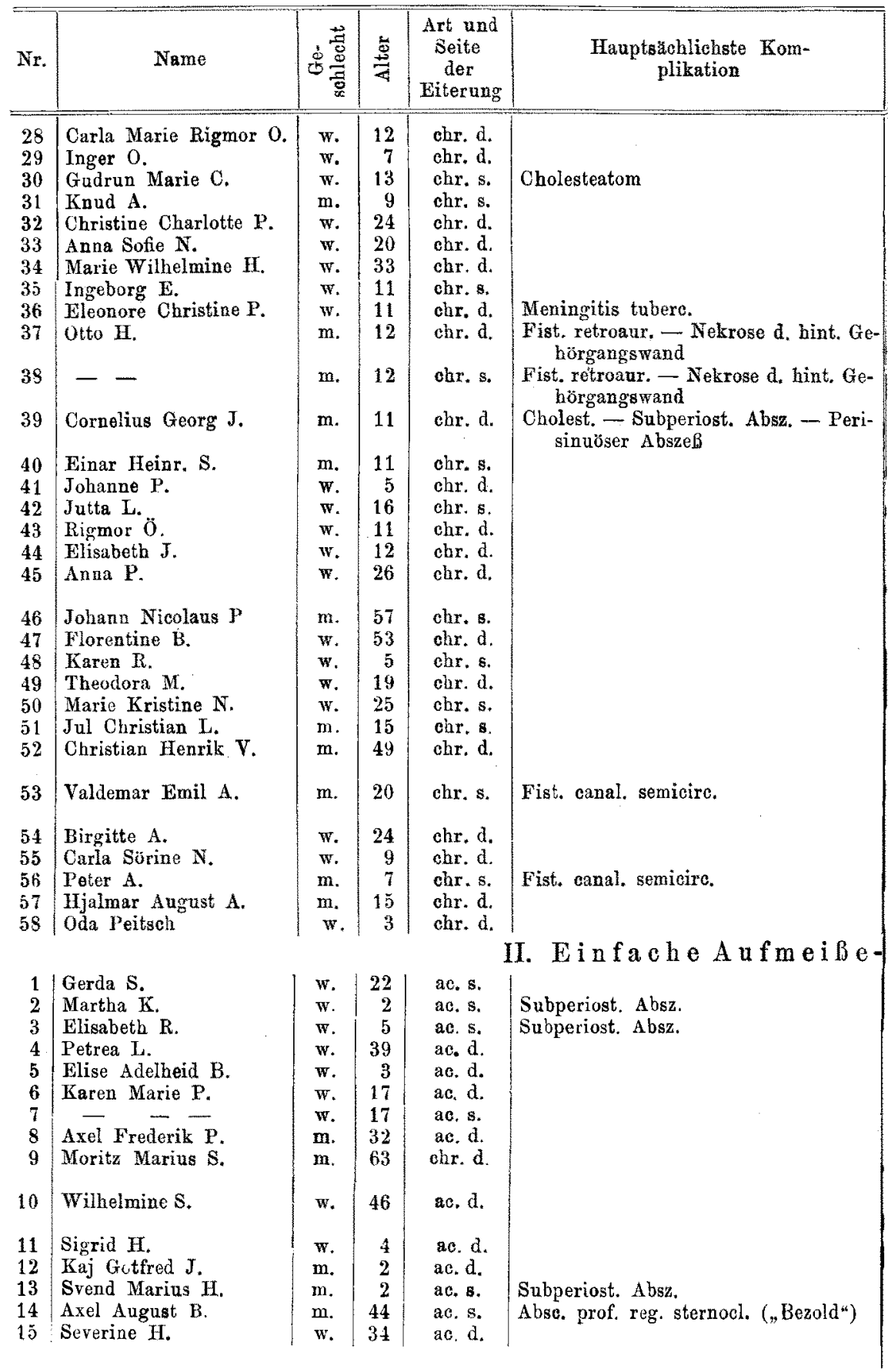


Jahresbericht des Kommunehospitals zu Kopenhagen.

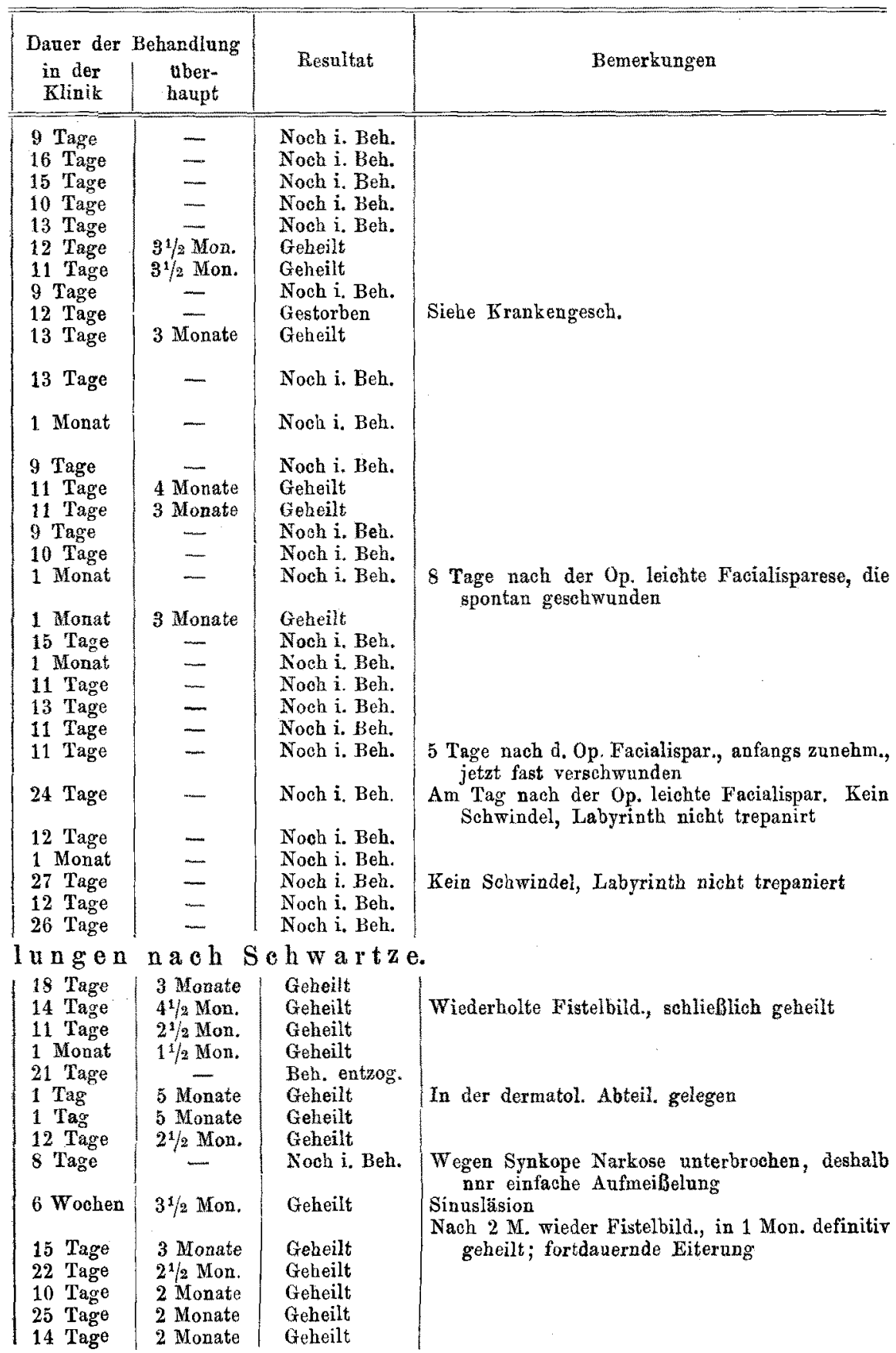




\begin{tabular}{|c|c|c|c|c|c|}
\hline Nr. & Name & 递 & 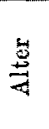 & $\begin{array}{l}\text { Art und } \\
\text { Seite } \\
\text { der } \\
\text { Eiterung }\end{array}$ & $\begin{array}{l}\text { Hauptsächlichste Kom- } \\
\text { plikationen }\end{array}$ \\
\hline 16 & Anders $J$ & $\mathrm{~m}$. & 2 & ac. s. & \\
\hline 17 & Emma $J$ & w. & 21 & ac. $d$. & \\
\hline 18 & Paula Johanna H. & w. & 4 & ac. d. & \\
\hline 19 & Graves Marinas $\mathrm{N}$. & m. & 4 & ac. d. & \\
\hline 20 & $\div--$ & m. & 4 & ac. $s_{*}$ & \\
\hline 21 & Kaj Christian P. & rn. & 1 & ac. $s$. & \\
\hline $\begin{array}{l}22 \\
23\end{array}$ & $\begin{array}{l}\text { Sören J. } \\
\text { Ellen Cathrine N. }\end{array}$ & $\begin{array}{l}\mathrm{m} . \\
\mathrm{w} .\end{array}$ & $\begin{array}{r}68 \\
5\end{array}$ & $\begin{array}{l}\text { ac, s. } \\
\text { ac, d. }\end{array}$ & \\
\hline 24 & Paula Johanne $H$. & w. & 4 & ac. s. & \\
\hline 25 & Servine Maria N. & w. & 39 & ac. s. & \\
\hline 26 & Faren Margretbe $J$. & w. & 1 & ac. s. & \\
\hline 27 & Julie $R$. & w. & 27 & ac. $s$ & \\
\hline 28 & Hans Christian $\mathrm{H}$. & $\mathrm{m}$. & 1 & ae. d. & Subperiost. Absz. \\
\hline 29 & Hedwig Dorthea P. & w. & 2 & ac. s. & Meningitis tub. - Miliärtub. \\
\hline 30 & Valdemar Angust M. & $\mathrm{m}$ & $5 !$ & ac. $\mathrm{s}$ & Sinusphlebit. - Neminglus. \\
\hline $\begin{array}{l}31 \\
32\end{array}$ & Henriette Johanne L. & w. & 33 & chr. d. & \\
\hline $\begin{array}{l}32 \\
33\end{array}$ & $\begin{array}{l}\text { Olga Amanda H, } \\
\text { Axel } 0 .\end{array}$ & $\begin{array}{l}\text { w. } \\
\text { m. }\end{array}$ & $\begin{array}{r}1 \\
13\end{array}$ & $\begin{array}{l}\text { ac. s. } \\
\text { ac. s. }\end{array}$ & Subperiost. Absz . \\
\hline $\begin{array}{l}34 \\
35\end{array}$ & $\begin{array}{l}\text { Sofie Magdalene } \mathrm{H} . \\
\text { Katbinka } \mathrm{N} \text {. }\end{array}$ & $\begin{array}{l}\text { w. } \\
\text { w. }\end{array}$ & $\begin{array}{l}52 \\
11\end{array}$ & $\begin{array}{l}\text { ac. d. } \\
\text { ae. \$. }\end{array}$ & $\begin{array}{l}\text { Eitr. Sinusthromb., Abszess, regs. ster- } \\
\text { noel. ("Bezold “?) }\end{array}$ \\
\hline 36 & Jensine $\mathrm{C}$ & w. & 30 & ac. s. & Absz. reg. sternocl. (,Bezold ${ }^{4}$ ?) \\
\hline 37 & Sophus Claudius $L$. & ma & 35 & ac, d. & \\
\hline 38 & Carl Ladwig B. & m. & 68 & ac. s. & \\
\hline $\begin{array}{l}39 \\
40\end{array}$ & $\begin{array}{l}\text { Anna } G \\
\text { Wilhelm } G \text {. }\end{array}$ & $\begin{array}{l}\text { w. } \\
\text { m. }\end{array}$ & $\begin{array}{r}3 \\
56\end{array}$ & $\begin{array}{l}\text { ac. d. } \\
\text { ac. d. }\end{array}$ & \\
\hline 41 & Hans Peter $J$ & $\mathrm{~m}$. & 54 & ac. d. & \\
\hline 42 & Erria R. & w. & 1 & ac. s. & \\
\hline
\end{tabular}
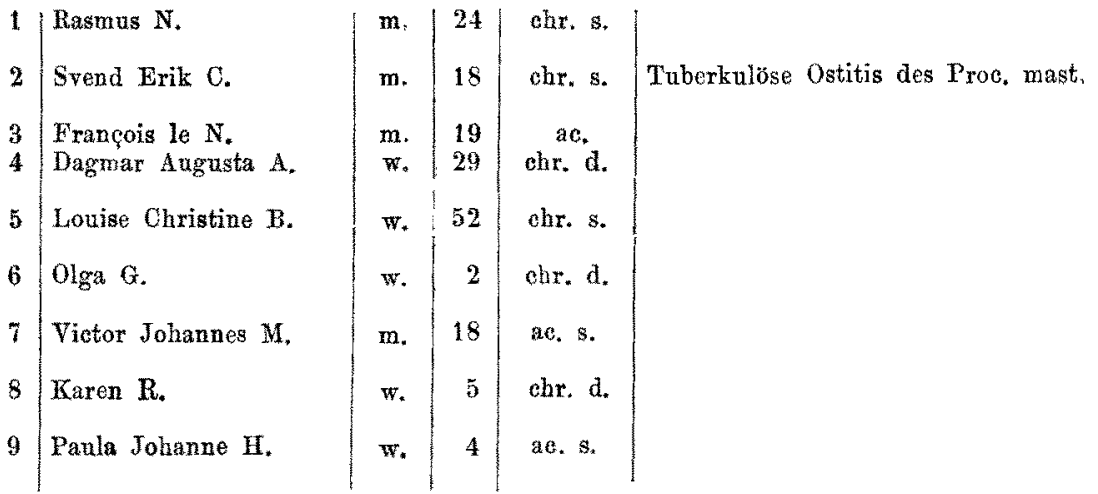


\begin{tabular}{|c|c|c|}
\hline $\begin{array}{c}\text { Dauer der } \\
\text { in der } \\
\text { Klinik }\end{array}$ & $\begin{array}{l}\text { Behandlung } \\
\text { iber- } \\
\text { haupt }\end{array}$ & Resultat \\
\hline 20 Tage & 7 Monat & Geheilt \\
\hline 19 Tage & 2 Monat & Geheilt \\
\hline 17 Tage & 2 Monat & Geheilt \\
\hline 1 Monat & 2 Monat. & Geheilt \\
\hline 1 Monat & 6 Woehen & Geheilt \\
\hline 1 Monat & - & Beh. entzog. \\
\hline 11 Tage & 3 Monate & Geheilt \\
\hline 19 Tage & 19 Tage & Geheilt \\
\hline 19 Tage & 19 Tage & Geheilt \\
\hline 1 Monat & 3 Monate & Geheilt \\
\hline 17 Tage & 17 Tage & Geheilt \\
\hline 11 Tage & 3 Monate & Geheilt \\
\hline 1 Nonat & $11 / 2$ Mon. & Geheilt \\
\hline 3 Tage & - & Gestorben \\
\hline 2 Tage & - & Gestorben \\
\hline $\begin{array}{l}12 \text { Tage } \\
7 \text { Wochen }\end{array}$ & $\begin{array}{l}21 / 2 \text { Mon. } \\
7 \text { Wochen }\end{array}$ & $\begin{array}{l}\text { Gebessert } \\
\text { Geheilt }\end{array}$ \\
\hline 10 Tage & 4 Monate & Geheilt \\
\hline 6 Woohen & - & Gestorben \\
\hline 4 Monate & - & Noch in Beh. \\
\hline 12 Tage & 2 Monate & Geheilt \\
\hline 17 Tage & 6 Wochen & Geheilt \\
\hline 1 Monat & 1 Monat & Geheilt \\
\hline 20 Tage & - & Beh. entz. \\
\hline 40 Tage & 2 Monate & Geheilt \\
\hline 21 Tage & - & Noch i. Beh. \\
\hline 22 Tage & - & Fast geheilt \\
\hline
\end{tabular}

A nfmeiBelungen.

\begin{tabular}{|c|c|c|c|}
10 Tage & 4 Monate & Geheilt \\
6 Woohen & 9 Monate & Geheilt \\
1 Tag & $3^{1 / 2}$ Mon. & Geheilt \\
22 Tage & 5 Monate & Geheilt \\
9 Tage & 6 Monate & Geheilt \\
7 Wochen & 7 Wochen & Geheilt \\
9 Tage & 3 Monate & Geheilt \\
1 Monat & - & Noch i. Beh. \\
16 Tage & - & Noch i. Beb.
\end{tabular}

1905 Totalaufm., nicht völl. epid., jetzt Cholest. u. Eit.; geöffnet u. revidiert

Vor 5 Jahr, ak. Mast, Aufm, geheilt; seit 3 Mon. Fistel. - Fistel geheilt, Eiterung sehr spärl.

3 M. fruher einf. Aufm, , immer Fistel, etw. Caries. Vor 3 Jahr, anderswo Totalaufm,, fortw. Eit; nicht weit genug geoffnet

Vor 5 Jahr. Totalaufm., geheilt. Jetzt 3 Mon. Eiterung; Diaphragmabildung

1906 Resekt., $4-5$ mal Wunde geheilt, wieder Fistelbild. - Naoh Revision defin. geheilt

Vor 1 Mon. im Auslande Resekt. Jetzt Fistel mit kaxiösem Knochen

Vor 3 Jahr. Totalaufm, retroaur. Oeffn, , fortw. Eit.; bedent. Caries $m$. Cholest.

$=$ einf. Aufm. N. 24. 1/2 Jahr nach d. Op. Abszeß-

bild. i. d. Narbe; Trommelfell naturlich

Primäre Nabt, 10 Tage nach d. Op. völlig geheilt Trommelfell nach 3 Wooh. geheilt. - Siebe doch atypische Aufmeiß Nr. 10

Siehe Krankengesch.

Wunde geheilt, fortdauernde geringe Eiterung

Wunde nach 2 Mon. geheilt, Eiterung nach 4 M. dauernde Perforation

Siebe Krankengesch.

Primäre Naht, sehr ausgedehnter ProzeB, Wunde mußte teilweise wieder geöfin. werden Eiter. i. d. Narbe, 3. Wooh. n. d. Op. alles heil.

Primäre Naht. Wunde nach 4 Tagen geheilt, Eiterung dauerte noch 4 Woch.

Prim. Naht, in $6 \mathrm{~T}$. scheinbar geh., später Fistelb. Wegen Tussis in d. epidem. Krankenhaus ubersied. 